Mots. Les langages du politique

\title{
Les débats de l'entre-deux-tours de l'élection présidentielle française (1974-2012) au prisme des stratégies discursives : du monopole du cœur à la doxa économico-comptable
}

Televised debates between France's presidential election rivals (1974-2012) and discourse strategies. Towards an accounting logic of the political issues Las estrategias discursives en los debates entre los dos turnos de las elecciones presidenciales francesas (1974-2012). Desde los sentimientos a la doxa económico-presupuestaria

Pierre-Olivier Dupuy et Pascal Marchand

\section{(2) OpenEdition} Journals

Édition électronique

URL : https://journals.openedition.org/mots/22472

DOI : $10.4000 /$ mots. 22472

ISSN : 1960-6001

Éditeur

ENS Éditions

Édition imprimée

Date de publication : 15 octobre 2016

Pagination : 69-80

ISBN : 978-2-84788-850-8

ISSN : 0243-6450

Référence électronique

Pierre-Olivier Dupuy et Pascal Marchand, « Les débats de l'entre-deux-tours de l'élection présidentielle française (1974-2012) au prisme des stratégies discursives : du monopole du cœur à la doxa économico-comptable », Mots. Les langages du politique [En ligne], 112 | 2016, mis en ligne le 15 octobre 2018, consulté le 23 avril 2022. URL : http://journals.openedition.org/mots/22472 ; DOI : https://doi.org/10.4000/mots.22472 


\section{Les débats de l'entre-deux-tours de l'élection présidentielle française (1974-2012) au prisme des stratégies discursives. Du monopole du cœur à la doxa économico-comptable}

«Haute tension». La Une du Figaro confirme, au lendemain du débat de l'entredeux-tours de l'élection présidentielle française de 2012 entre François Hollande et Nicolas Sarkozy, que ce rituel médiatico-politique de la Cinquième République est perçu, dans les représentations collectives, comme une arène où s'opposent et s'affrontent deux duellistes, candidats à la présidence. Si les champs lexicaux du combat et du duel sont convoqués lors des analyses pré- et post-débat par les éditorialistes et personnalités politiques (Dupuy, 2008; Dupuy, Marchand, 2009), il est peu fait état de la tension interne vécue par chaque codébatteur lors de cet événement très attendu. Car, dans cette situation de communication bien particulière ${ }^{1}$, les deux finalistes se trouvent dans une problématique identitaire complexe. Il convient tour à tour d'incarner un parti, une idéologie et un électorat en se construisant un ethos positif (Amossy, 1999), au travers d'une identité lexicale et discursive construite et répétée durant la campagne, tout en essayant de convaincre des catégories d'électeurs non acquis (membres d'exogroupes : électeurs hostiles et indécis notamment) de voter pour soi (ou contre son adversaire). Cet exercice est d'autant plus délicat qu'au-delà du temps imparti par le format (entre deux et trois heures de débat télévisé), chaque candidat vise ce même objectif au sein d'un contrat de communication qui autorise interruptions, chevauchements de

Ce travail a été réalisé dans le cadre du LabEx Structuration des mondes sociaux (SMS) portant la référence ANR-11-LABX-0066.

1. Il s'agit de l'unique face-à-face opposant, pour la première fois de la campagne, les deux candidats choisis à l'issue du premier tour de vote, animé par deux journalistes-métronomes (fonction dévolue de respect du temps de parole) et dont le dispositif sémio-discursif (choix des plans et des thématiques principalement) est cadré en amont par les équipes de chaque candidat. Ce dispositif le distingue ainsi des autres débats électoraux et télévisés : émissions politiques, talk-shows, etc. (voir Kerbrat-Orrechioni, 2013).

Université Paul Sabatier-Toulouse 3, LERASS, équipe Psycom pierre-olivier.dupuy@iut-tarbes.fr

Université Paul Sabatier-Toulouse 3, LERASS, équipe Psycom

pascal.marchand@iut-tlsez.fr 
discours et interpellations directes de son adversaire. Ainsi, dans une approche psychosociale et interactionnelle (Goffman, 1973; Mead, 2006; Deschamps, Moliner, 2008), nous analysons comment se négocie la construction identitaire opérée par les codébatteurs : par quelles stratégies discursives les duellistes cherchent-ils à incarner et attirer une majorité d'électeurs? Comment passer de la représentation d'un infragroupe politique (appartenir à un parti et avoir une place déterminée sur l'échiquier politique national) à celle recherchée d'un supragroupe? Comment, dans l'histoire de la Cinquième République, cette problématique identitaire a-t-elle évolué et que nous apprend-elle de l'évolution des discours des présidentiables?

\section{La campagne présidentielle : un duel de stratégies discursives}

L'élection présidentielle est une succession d'étapes discursives qui mobilisent des enjeux identitaires distincts. Les mois précédant la présidentielle permettent aux personnalités politiques en quête de notoriété et de valorisation de multiplier les plateaux de télévision au sein d'émissions diverses ou lors de sorties en librairie de livres politiques (Leroux, Riutort, 2014). L'étape des «primaires» relève davantage d'une dynamique intragroupe et consiste, pour chacun des candidats à l'investiture d'un parti, à "convaincre de ses similitudes [intragroupes] tout en démontrant ses différences [interpersonnelles]» (Mange, Marchand, 2011, p. 87; Marchand, Ratinaud, 2014). Le temps de la campagne est, quant à lui et jusqu'au premier tour, celui d'une différenciation intergroupe qui accentue les clivages politiques, chaque parti en lice cherchant à rassembler autour d'un programme et d'une personnalité, à minimiser les tensions en interne et à se démarquer des propositions, des partis et des personnalités consacrées adverses : s'observent alors une maximisation des différences intergroupes mais aussi interpersonnelles avec les candidats des exogroupes, en tentant d'édifier sa position de présidentiable au gré des représentations médiatiques (Garcin-Marrou, 2009; Le Bart, 2009; Baider, 2015). Or, si jusqu'à présent nous retrouvons les processus inhérents à la construction d'une identité sociale de la part des candidats (nous vs eux; moi, candidat ou candidate à la présidence $v s$ les autres candidates ou candidats; Tajfel, Turner, 1986; Deschamps, Moliner, 2008; Dupuy, 2008), le deuxième tour représente une étape et une stratégie encore différentes où chaque «finaliste » doit dépasser ses appartenances groupales : il s'agit en effet de fédérer certains groupes adverses du premier tour (y compris dans son propre camp dans une vision bipartite de l'offre politique : gauche vs droite), de se différencier de son adversaire et de tenter de présenter un rassemblement plus large encore de tous les électeurs potentiels. Au-delà de ces aspects identitaires, chacun doit également faire preuve de sa compétence à gouverner, valorisant 
son bilan, atténuant ses échecs, développant un projet crédible et attractif. Les lexiques mobilisés sont donc tributaires, à la fois, de ces enjeux identitaires et programmatiques, mais aussi des caractéristiques situationnelles du débat (thématiques conjoncturelles) et de l'expérience personnelle du candidat. Or, dans une perspective de rassemblement maximisée, chaque duelliste prend le risque, à chercher l'ouverture à d'autres thématiques et sensibilités politiques, de s'immiscer sur un terrain qu'il maitrise moins, ou marqué par le sceau de son adversaire, ou bien encore de commettre, par dialogisme, des incohérences interdiscursives ${ }^{2}$. Si l'histoire a davantage retenu les petites phrases et acmés dramaturgiques des différents débats ${ }^{3}$, cette tension identitaire propre à ce débat télévisé a aussi engendré, en trente ans, un glissement dans le contenu des discours des codébatteurs au sein desquels la polémique et la gestion comptable de la société ont pris le dessus sur les projets politiques.

\section{Évolutions thématiques : la rupture des années 2000}

Dans le cadre de nos analyses textométriques4, l'intégralité des six débats de l'entre-deux-tours a été saisie5 : 10 mai 1974 (Valéry Giscard d'Estaing et François Mitterrand), 5 mai 1981 (Valéry Giscard d'Estaing et François Mitterrand), 28 avril 1988 (Jacques Chirac et François Mitterrand), 2 mai 1995 (Jacques Chirac et Lionel Jospin), 2 mai 2007 (Ségolène Royal et Nicolas Sarkozy), 2 mai 2012 (François Hollande et Nicolas Sarkozy). À noter, l'absence de débat en 2002, Jacques Chirac refusant de débattre avec Jean-Marie Le Pen. Le corpus a notamment été codé avec la variable de l'année permettant une analyse diachronique des pratiques langagières des prétendants à l'Élysée (Dupuy, Marchand, 2011). Il est ainsi composé de 2313 tours de parole, représentant 149060 occurrences qui mobilisent un lexique de 8923 formes ( 5266 après lemmatisation). Une analyse des correspondances permet d'identifier une distribution chronologique des débats de l'entre-deux-tours (figures 1 et 2).

2. Que ne manqueront pas de relever leur adversaire sur le moment, ou les commentateurs dès le lendemain du débat, le web permettant aisément de retrouver les discours des uns et des autres et de les partager.

3. Certaines phrases, attaques et épisodes sont ainsi devenus emblématiques d'un débat. C'est le cas des expressions suivantes : "monopole du cœur» (Valéry Giscard d'Estaing à François Mitterrand, 1974); "Mais vous avez tout à fait raison, monsieur le premier ministre» (François Mitterrand, en réponse à Jacques Chirac qui venait de lui dire : "Ce soir, vous n’êtes pas le président de la République, nous sommes deux candidats à égalité [...], vous me permettrez donc de vous appeler monsieur Mitterrand », 1988) ; l'épisode de la « saine colère » de Ségolène Royal (2007) ; l’anaphore «Moi, président de la République » de François Hollande (2012)...

4. Avec le logiciel libre Iramuteq développé par Pierre Ratinaud au sein du LERASS (EA 827, Université Paul Sabatier-Toulouse 3) grâce au soutien du Labex SMS (Université de Toulouse).

5. Pour une description des choix méthodologiques de constitution du corpus et des analyses, voir Dupuy, Marchand, 2013. 


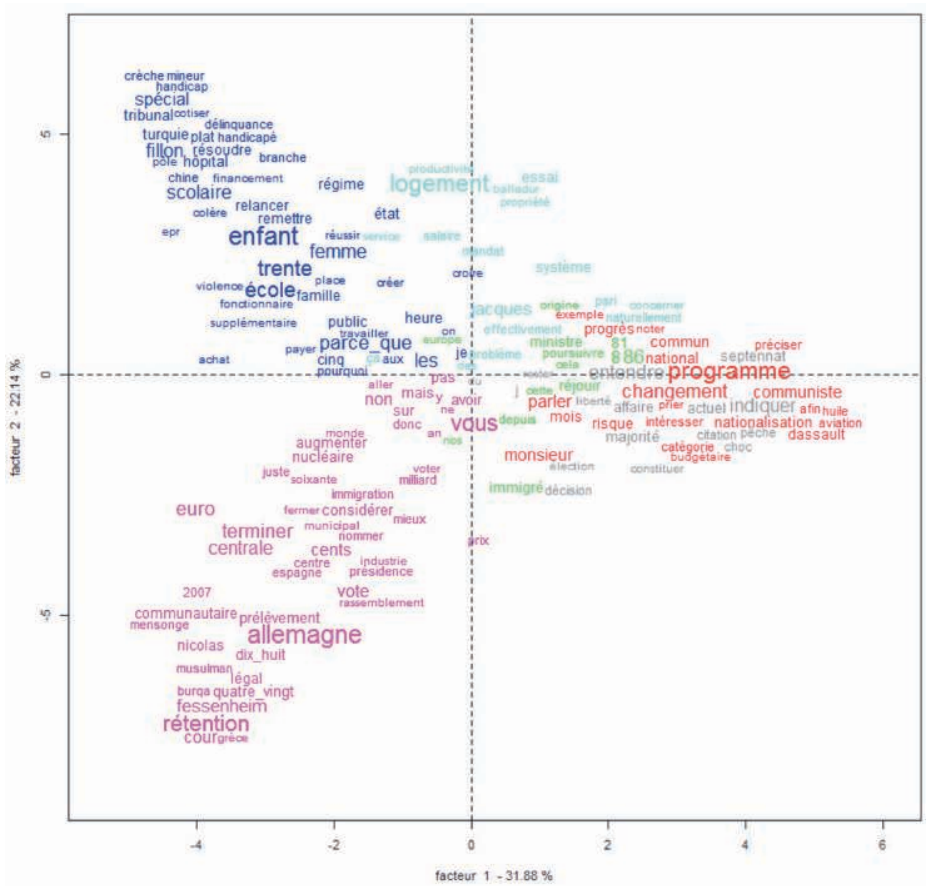

Figure 1 : AFC des débats (lexique)

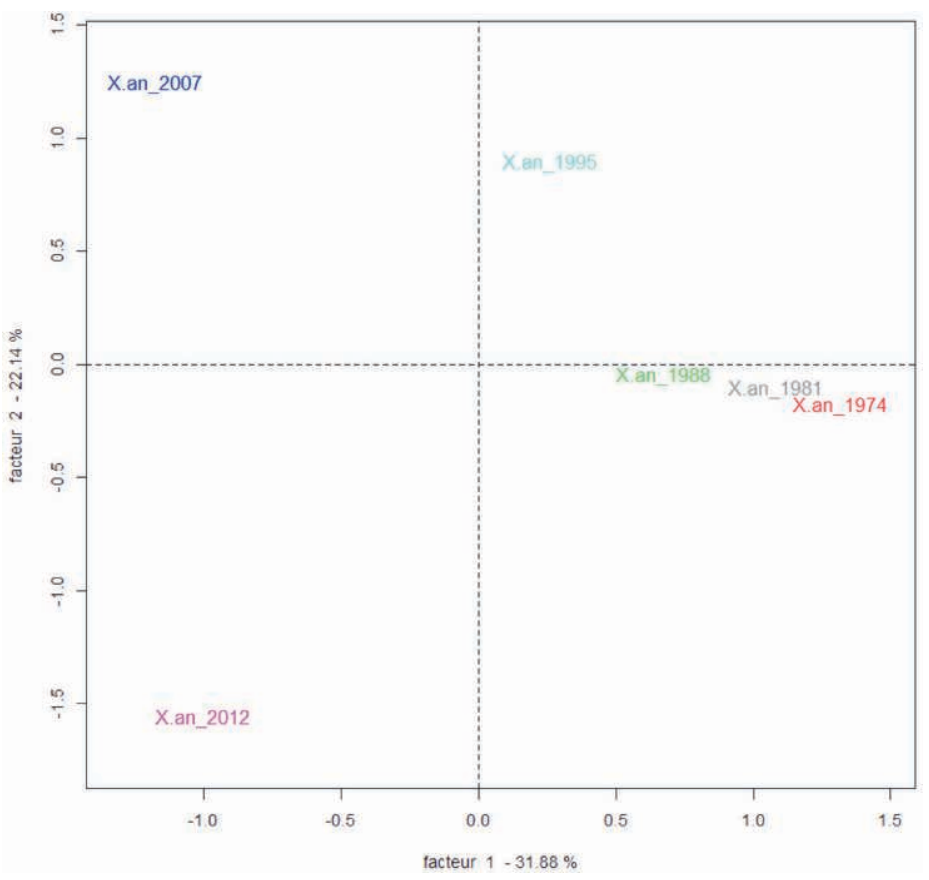

Figure 2 : AFC des débats (années) 
Le plan factoriel indique (de droite à gauche) une proximité des lexiques lors des trois premiers débats (1974-1988) et un début de différenciation avec celui de 1995. En revanche, les deux derniers débats (2007 et 2012) apparaissent en rupture. Une classification lexicale permet de mieux décrire cette dynamique.

Le corpus a été partitionné en 5475 segments de textes (de vingt-sept formes en moyenne) pour pouvoir faire l'objet d'une classification descendante hiérarchique (CDH, méthode Reinert, 1983). L'analyse permet de définir dix-sept classes lexicales, dont le dendrogramme est présenté figure 3 (pages suivantes).

S'il n'est pas possible de décrire précisément ici toutes ces classes, nous pouvons nous laisser guider par la chronologie pour définir des tendances historiques.

La figure 4 (pages suivantes) permet de retrouver et de préciser les résultats suggérés par l'analyse factorielle des correspondances (AFC).

Certaines thématiques apparaissent peu sensibles à la chronologie, et représentent des «passages obligés» qui tiennent éventuellement davantage à la forme qu'au fond. Sans surprise, compte tenu de la situation de débat électoral, nous retrouvons ainsi plusieurs champs lexicaux qui renvoient à l'acte langagier: prendre position, affirmer une opinion, expliquer (Classe $6: 9,5 \%$ ) ; répondre à la question, débattre (Classe 9:4\%) ; s'engager (Classe 8:4,3\%). Se référer aux institutions (Classe 15:1,8\%) et promouvoir l'histoire et l'image de la France dans le monde (Classe $5: 3,5 \%$ ) relèvent également d'une continuité dans le temps.

Un groupe de thématiques semble cependant marquer davantage le début de la période et est caractérisé par la forte thématique des appartenances politiques, de la gouvernance à venir et des projets politiques (Classe $3:$ le gouvernement, le parlement, 5,6\%; Classe 4 : l'exécutif et les partis politiques, $7,2 \%$; Classe 7 : les projets, l'avenir, 9,4\%; Classe 12 : l'Europe, 7,2 \% à partir de 1981). Nous observons ensuite une liaison particulière de la Classe 13 avec le débat de 1995 : la thématique du service public et de son financement. Cette thématique est également reprise, mais moins significativement, en 2007 (voir Dupuy, 2008), année au cours de laquelle la rupture observée sur les AFC se confirme ici en mobilisant les classes 10 (les retraites : 7,5\%), 11 (l'entreprise, le temps de travail, l'emploi et la question des salaires : $10 \%$ ) et surtout 16 (la question de l'enfance, de l'école et du handicap : 3,4\%)6. Lors du débat de 2012, ce sont des thématiques très spécifiques qui sont mobilisées : la crise économique et la question des déficits occupent le premier tiers du débat (Classe $1: 6,2 \%$ ), et les codébatteurs échangent particulièrement sur trois thèmes : le pouvoir d'achat (Classe $2: 3,8 \%$ ), l'immigration (Classe 14 : $5,4 \%$ ) et le nucléaire (Classe $17: 2,9 \%$ ).

6. À l'intérieur de laquelle se situe l'épisode devenu célèbre de la « saine colère ». Il s'agit également du seul débat de l'entre-deux-tours qui a vu s'opposer une candidate et un candidat (Voir Kerbrat-Orrechioni, 2013). 


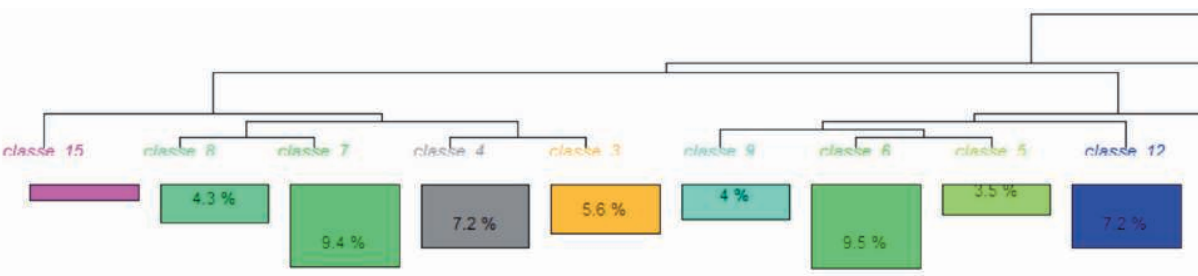

\begin{tabular}{|c|c|c|c|c|c|c|c|c|}
\hline $\begin{array}{l}\text { gaulle } \\
\text { général } \\
\text { magistrature } \\
\text { georges } \\
\text { pormpidou } \\
\text { rue } \\
\text { violer } \\
\text { prison } \\
\text { mort } \\
\text { valéry } \\
\text { policier } \\
\text { lettre } \\
\text { consel } \\
\text { supérieur } \\
\text { magistrat } \\
\text { tromper } \\
\text { remarquable } \\
\text { parquet } \\
\text { siége } \\
\text { ope } \\
\text { collègue } \\
\text { offioiel } \\
\text { conseller } \\
\text { réunion } \\
\text { gobal } \\
\text { préfet } \\
\text { invoquer }\end{array}$ & $\begin{array}{l}\text { décentralisi } \\
\text { information } \\
\text { important } \\
\text { sortir } \\
\text { multiplier } \\
\text { page } \\
\text { doute } \\
\text { chef } \\
\text { récemment } \\
\text { etat } \\
\text { statut } \\
\text { raison } \\
\text { nouvelle } \\
\text { journal } \\
\text { probablement } \\
\text { désagréable } \\
\text { profond } \\
\text { campagne } \\
\text { reconnalire } \\
\text { souftir } \\
\text { coent } \\
\text { téférendum } \\
\text { tooutes } \\
\text { président }\end{array}$ & $\begin{array}{l}\text { rassemblen } \\
\text { responsabil } \\
\text { avenir } \\
\text { intéresser } \\
\text { débat } \\
\text { français } \\
\text { assumer } \\
\text { sujet } \\
\text { parler } \\
\text { esprit } \\
\text { homme } \\
\text { engager } \\
\text { choix } \\
\text { gauche } \\
\text { tour } \\
\text { candidat } \\
\text { soir } \\
\text { électeur } \\
\text { république } \\
\text { confiance } \\
\text { trancois } \\
\text { tadairer } \\
\text { dimanche } \\
\text { rejoindie }\end{array}$ & $\begin{array}{l}\text { président } \\
\text { république } \\
\text { majorité } \\
\text { ministré } \\
\text { élire } \\
\text { nommer } \\
\text { communiste } \\
\text { député } \\
\text { élection } \\
\text { présidence } \\
\text { gouvernement } \\
\text { lègisiatif } \\
\text { représentation } \\
\text { finanoe } \\
\text { mai } \\
\text { cas } \\
\text { socialste } \\
\text { premier } \\
\text { personnalité } \\
\text { commission } \\
\text { partir } \\
\text { compte } \\
\text { consel } \\
\text { époquet } \\
\text { actuel } \\
\text { dissolution } \\
\text { formes }\end{array}$ & 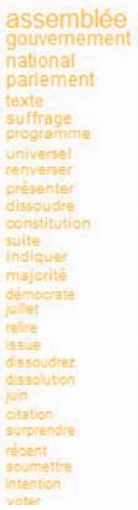 & 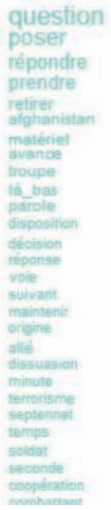 & $\begin{array}{l}\text { penser } \\
\text { problème } \\
\text { politique } \\
\text { chose } \\
\text { distance } \\
\text { voir } \\
\text { aller } \\
\text { bouger } \\
\text { différence } \\
\text { reprendre } \\
\text { russie } \\
\text { préferer } \\
\text { simple } \\
\text { priconiser } \\
\text { protestation } \\
\text { institutionnel } \\
\text { axee } \\
\text { expliquer } \\
\text { arritter } \\
\text { comprendre } \\
\text { passion } \\
\text { enrichissement } \\
\text { ambition } \\
\text { changement } \\
\text { action } \\
\text { nisonnement } \\
\text { porte }\end{array}$ & $\begin{array}{l}\text { histoire } \\
\text { force } \\
\text { partager } \\
\text { tchad } \\
\text { rang } \\
\text { échéance } \\
\text { regretter } \\
\text { affaire } \\
\text { libye } \\
\text { faille } \\
\text { france } \\
\text { combattre } \\
\text { sentiment } \\
\text { centrafrique } \\
\text { morai } \\
\text { période } \\
\text { cause } \\
\text { risque } \\
\text { principe } \\
\text { eprouver } \\
\text { porner } \\
\text { eloigner } \\
\text { industoiel } \\
\text { exdure } \\
\text { poids }\end{array}$ & $\begin{array}{l}\text { europe } \\
\text { turquie } \\
\text { union } \\
\text { communauté } \\
\text { commun } \\
\text { défense } \\
\text { international } \\
\text { conséquence } \\
\text { soviétique } \\
\text { désarmement } \\
\text { bretagne } \\
\text { entrée } \\
\text { rome } \\
\text { pêche } \\
\text { changer } \\
\text { marché } \\
\text { élargissement } \\
\text { peuple } \\
\text { démocratique } \\
\text { contrôle } \\
\text { référendum } \\
\text { défendre } \\
\text { avis } \\
\text { européen } \\
\text { possible }\end{array}$ \\
\hline
\end{tabular}

Figure 3 : Dendrogramme de la CDH des débats (Iramuteq)

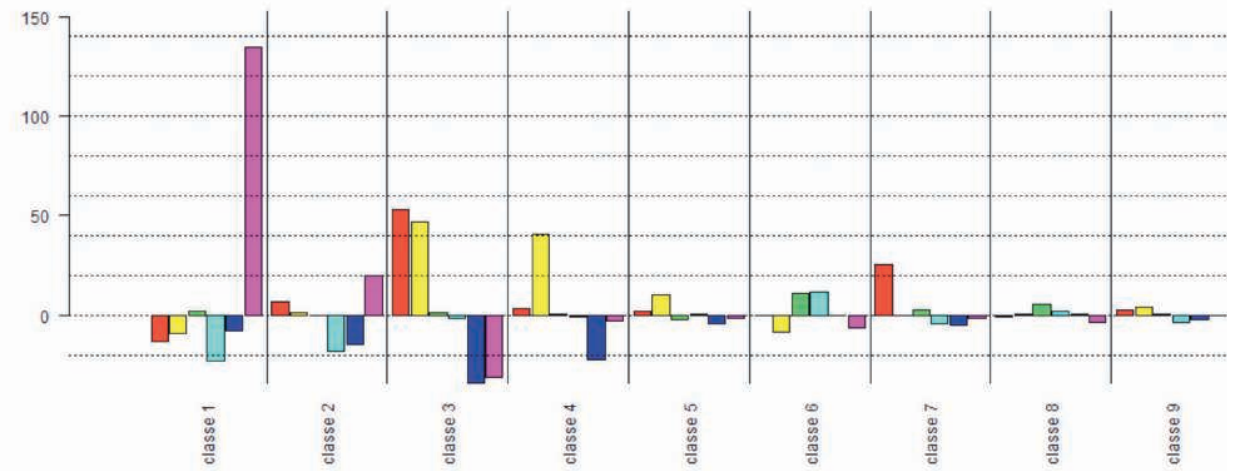

Figure 4 : Projection de la chronologie sur le dendrogramme de la CDH 


\begin{tabular}{|c|c|c|c|c|c|c|c|}
\hline rlascle 14 & rlacde 17 & dact $\bar{C}$ ? & classe 1 & clacde 13 & clacde 11 & rianes in & riacda 16 \\
\hline $5.4 \%$ & & $3.8 \%$ & $6.2 \%$ & $82 \%$ & & $.5 \%$ & $1.4 \%$ \\
\hline $\begin{array}{l}\text { étranger } \\
\text { vote } \\
\text { droit } \\
\text { immigration } \\
\text { clandestin } \\
\text { loi } \\
\text { communautain } \\
\text { musulman } \\
\text { voile } \\
\text { appliquer } \\
\text { immigré } \\
\text { uniquement } \\
\text { séjour } \\
\text { burqa } \\
\text { illégal } \\
\text { légal } \\
\text { nationalité } \\
\text { municipal } \\
\text { femme } \\
\text { code } \\
\text { migratoire } \\
\text { flux } \\
\text { apprendre } \\
\text { naite } \\
\text { voler } \\
\text { papier }\end{array}$ & $\begin{array}{l}\text { centrale } \\
\text { fermer } \\
\text { vieux } \\
\text { nucléaire } \\
\text { fessenheim } \\
\text { finlande } \\
\text { epr } \\
\text { intelligent } \\
\text { fukushima } \\
\text { sismique } \\
\text { renouvelable } \\
\text { islande } \\
\text { génération } \\
\text { vendre } \\
\text { géner } \\
\text { quatrième } \\
\text { prolonger } \\
\text { joly } \\
\text { réacteur } \\
\text { construire } \\
\text { confirmer } \\
\text { essai } \\
\text { récossion } \\
\text { choó } \\
\text { zone } \\
\text { vert } \\
\text { aocord } \\
\text { ant }\end{array}$ & $\begin{array}{l}\text { prix } \\
\text { électricité } \\
\text { acheter } \\
\text { pétrole } \\
\text { gaz } \\
\text { blocage } \\
\text { inflation } \\
\text { consommateur } \\
\text { produit } \\
\text { tva } \\
\text { budgétaire } \\
\text { huile } \\
\text { distribution } \\
\text { essence } \\
\text { coúter } \\
\text { cher } \\
\text { atteindre } \\
\text { équivalent } \\
\text { alimentaire } \\
\text { millard } \\
\text { honnêtement } \\
\text { déficit } \\
\text { nuciéaire } \\
\text { mois } \\
\text { renouvelabies } \\
\text { enrergie } \\
\text { priter } \\
\text { espace }\end{array}$ & $\begin{array}{l}\text { milliard } \\
\text { euro } \\
\text { allemagne } \\
\text { impôt } \\
\text { déficit } \\
\text { prélèvement } \\
\text { obligatoire } \\
\text { riche } \\
\text { fortune } \\
\text { taxe } \\
\text { augmenter } \\
\text { faute } \\
\text { transaction } \\
\text { financier } \\
\text { dégradé } \\
\text { structurel } \\
\text { danemark } \\
\text { orise } \\
\text { chiffre } \\
\text { commerce } \\
\text { pays } \\
\text { etats } \\
\text { unir } \\
\text { élevé } \\
\text { remarquer } \\
\text { dwuhis }\end{array}$ & $\begin{array}{l}\text { public } \\
\text { fonctionnaire } \\
\text { logement } \\
\text { service } \\
\text { crédit } \\
\text { dette } \\
\text { fonction } \\
\text { dépenser } \\
\text { argent } \\
\text { propriétaire } \\
\text { propriété } \\
\text { banque } \\
\text { prêt } \\
\text { him } \\
\text { hospitalier } \\
\text { dépense } \\
\text { etat } \\
\text { acoéder } \\
\text { rembourser } \\
\text { budget } \\
\text { investissement } \\
\text { emprunt } \\
\text { pari } \\
\text { nuit } \\
\text { fonds } \\
\text { assurance } \\
\text { perte }\end{array}$ & $\begin{array}{l}\text { entreprise } \\
\text { salaire } \\
\text { heure } \\
\text { emploi } \\
\text { durée } \\
\text { cotisation } \\
\text { travail } \\
\text { supplémentaire } \\
\text { jeune } \\
\text { charge } \\
\text { smic } \\
\text { embauoher } \\
\text { moyen } \\
\text { petit } \\
\text { employeut } \\
\text { augmentation } \\
\text { salarié } \\
\text { payer } \\
\text { partenaire } \\
\text { investir } \\
\text { crees } \\
\text { donner } \\
\text { diminuer } \\
\text { permettre } \\
\text { syndicat } \\
\text { environnement } \\
\text { utiliser } \\
\text { indéterminé }\end{array}$ & 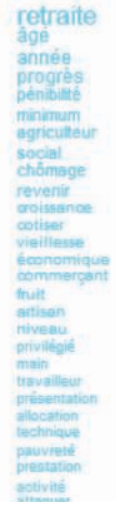 & $\begin{array}{l}\text { enfant } \\
\text { école } \\
\text { scolaire } \\
\text { élève } \\
\text { enseignant } \\
\text { handicapé } \\
\text { établissement } \\
\text { faux } \\
\text { professeur } \\
\text { collêge } \\
\text { rentrer } \\
\text { acoueillir } \\
\text { primaire } \\
\text { scolarisès } \\
\text { parent } \\
\text { soutien } \\
\text { individuelement } \\
\text { adulte } \\
\text { handicap } \\
\text { supprimer } \\
\text { enseignement } \\
\text { poste } \\
\text { maternel } \\
\text { noyer } \\
\text { martine } \\
\text { résoudre } \\
\text { classe } \\
\text { ditunatair }\end{array}$ \\
\hline
\end{tabular}

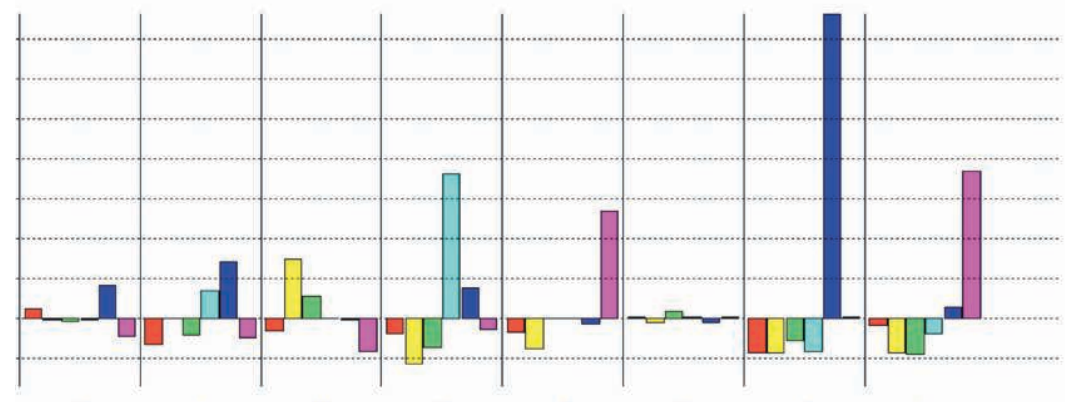




\section{Discussion}

Si, bien entendu, certaines thématiques sont conjoncturelles (comme les questions internationales souvent reléguées en fin de débat ou l'importance accordée à la crise économique en 2012 : Dupuy, Marchand, 2013), des dynamiques plus profondes sont à l'œuvre, marquant les stratégies diachroniques de construction d'identité des duellistes. Ainsi, le début de période (1974 à 1988) voit s'affronter des candidats aux identités politiques fortes, marquées parl'affirmation des appartenances partisanes et la représentation d'un groupe politique. Le pronom «nous», marqueur langagier englobant un parti et une équipe derrière chaque duelliste, laisse place au fil des décennies à l'usage massif du pronom «je» (Calvet, Véronis, 2008; Dupuy, Marchand, 2011), centre d'une identité personnelle et de l'affirmation émotionnelle (Kerbrat-Orecchioni, 2010). Les rhétoriques affectives sont en effet de plus en plus utilisées et le rapprochement avec les citoyens recherché, d'autant que le recours croissant au pronom indéterminé « on » permet à chaque candidat de se déguiser tour à tour en chômeur, mère de famille, dirigeant d'entreprise, travailleur du quotidien (débats de 2007 et 2012). La double énonciation à l'œuvre met en exergue cette quête identitaire maximisée à quelques jours du second tour : se distinguer drastiquement de l'autre (je, moi vs vous), mettre en avant sa singularité, ses compétences et ses valeurs tout en subsumant son identité politique marquée sur l'échiquier de l'offre politique en lice et devenir le candidat rassembleur parlant au nom de tous (on, nous). La recherche de cette incarnation passe également par une objectivation des prises de position et des représentations simplifiées d'une réalité par nature pourtant complexe : question rhétorique, recours aux marqueurs d'existence (il y $a$, il faut), syllogismes et conclusions présentées comme inéluctables, et apologie du chiffre, comme dans cette démonstration du candidat Sarkozy (en 2012) usant d'effet de scientificité : «Prenons les chiffres parce que c'est important pour ceux qui nous écoutent, du BIT. C'est un chiffre incontestable. Le chômage a augmenté de 422000 , ce qui est trop entre 2007 et 2011, chiffre BIT [...] Mes solutions pour l'avenir. Premièrement, il y a un gigantesque problème de coût du travail [...] Il faut alléger le coût du travail».

Les projections sur les modalités de gouvernance et les visions politiques sont petit à petit remplacées par un vocabulaire économique et comptable de plus en plus présent, sur la base de comparaisons avec d'autres pays pris pour modèles, de statistiques avancées et d'indicateurs normés (Gobin, 2002 ; Lallement, Spurk, 2003; Angeletti, 2011)7 : le chiffre apparait alors comme un moyen de dépassement du discours idéologique pourviser l'objectivité transversale au

7. Voir également R. Cussó et C. Gobin (2008). 
groupe (Desrosières, 2008; Ogien, 2010) ${ }^{8}$, offrant ainsi en spectacle la lutte pour s'arroger la vérité («mon chiffre est bon, pas le vôtre»). Stratégie des équipes de communicants ou amoindrissement d'une parole politique à même de construire un projet politique visionnaire, la disparition des questions politiques s'accompagne d'une banalité lexicale en hausse : chacun puise dans un discours de plus en plus pauvre, commun et redondant, dans une quête de répétition, empruntée au marketing, de ses propres mots, de ses slogans et de son catalogue de mesures et dans une quête potentielle de proximité avec l'ensemble des électeurs.

La recherche d'une maximisation de la représentation d'une majorité d'électeurs amène, dans un contexte d'altération politique (Linhardt, Muniesa, 2011), une perte de repères idéologiques forts, marqués par des appartenances groupales assumées, au profit d'un discours quantifié (quel que soit le sujet abordé), pragmatique et dépolitisé, valorisant, d'une part, les compétences gestionnaires des codébatteurs et, d'autre part, leur identité personnelle, leurs considérations morales (courage/lâcheté; vérité/mensonge) et leur capacité à s'émouvoir de situations vécues par certaines catégories sociales. Absents du débat en raison du dispositif mis en place, les citoyens-téléspectacteurs observent alors un dialogue de «parler vrai» (Oustinoff, 2010; Planchenault, 2014), en quête de présidentialisation, sans qu'experts, scientifiques, journalistes ou acteurs économiques et sociaux ne puissent s'interposer légitimement dans cet entre-soi de personnalités. Dans cette quête identitaire et ce jeu de négociations qu'est le discours politique (Amossy, 1999; Mayaffre, 2003; Charaudeau, 2005), et en sus de la mise en exergue des thématiques9 et des évolutions langagières permise par ces analyses, force est de constater que disparait au fil de ces débats télévisuels électoraux la monstration d'une capacité à penser à long terme l'action politique, ancrée dans un projet et une idéologie politiques ${ }^{10}$, sans que les effets d'objectivation et de proximisation ne semblent altérer une méfiance accrue envers les élites, un absentéisme électoral prégnant et une déception à l'égard des responsables politiques qui touche également les partisans de chaque formation au pouvoir.

\section{Références}

Amossy Ruth, 1999, Images de soi dans le discours. La construction de l'ethos, Lausanne, Delachaux et Niestlé.

ANGELLETTI Thomas, 2011, «Faire la réalité ou s’y faire? La modélisation et les déplacements de la politique économique au tournant des années 1970 », Politix, n²95, p. 47-72.

8. Voir également P. Bacot, D. Desmarchelier et S. Rémi-Giraud (2012).

9. Voir également M. Ben Hamed et D. Mayaffre (2015).

10. Dynamique qui se confirme généralement, quelques semaines après l'élection du président, dans les déclarations de politique générale des Premiers ministres (Marchand, 2007). 
Bacot Paul, Desmarchelier Dominique et Rémi-Giraud Sylvianne éd., 2012, Mots. Les Langages du politique, $\mathrm{n}^{\circ} 100$, «Chiffres et nombres dans l'argumentation ».

BAIDER Fabienne H., 2015, "Évaluation de la présidentiabilité (à l'interface de la sémantique lexicale et de la linguistique de corpus) », Mots. Les langages du politique, n 108, "Thèmes et thématiques dans le discours politique », p. 103-128.

Ben Hamed Mahé et Mayaffre Damon éd., 2015, Mots. Les Langages du politique, $\mathrm{n}^{0} 108$, "Thèmes et thématiques dans le discours politique ».

CAlVet Louis-Jean, Véronis Jean, 2008, Les mots de Nicolas Sarkozy, Paris, Le Seuil.

Chauradeau Patrick, 2005, Le discours politique. Les masques du pouvoir, Paris, Vuibert.

Cussó Roser et Gobın Corinne éd., 2008, Mots. Les Langages du politique, n88, « Du discours politique au discours expert. Le changement politique mis hors débat?».

Deschamps Jean-Claude, Moliner Pascal, 2008, L'identité en psychologie sociale, Paris, Armand Colin.

Desrosières Alain, 2008, L'argument statistique, t. l, Pour une sociologie historique de la quantification, t.Il, Gouverner par les nombres, Paris, Mines-ParisTech-Les Presses.

Dupuy Pierre-Olivier, 2008, L'interaction politique vue comme un processus de construction identitaire: le cas des présidentielles 2007. Analyse du débat de l'entre-deux-tours et des questions de citoyens, Thèse de Doctorat en sciences de l'information et de la communication, Université Paul Sabatier-Toulouse 3.

Dupuy Pierre-Olivier, MARChAnd Pascal, 2009, «Débat de l'entre-deux-tours. La conquête de l'espace lexical», Mots. Les langages du politique, n॰89, « 2007. Débats pour l'Élysée », p.105-117.

- 2011, "Confrontation et positionnement dans les duels de l'entre-deux-tours. Une approche lexicométrique", La parole politique en confrontation dans les médias, M. Burger, J. Jacquin, R. Micheli éd., Bruxelles, De Boeck, p. 131-150.

- 2013, "Évolutions lexicales des débats de l'entre-deux-tours de l'élection présidentielle française. Du discours de la quantification à la polémique, des stratégies argumentatives au service des électeurs? ", Bulletin suisse de linguistique appliquée, $\mathrm{n}^{\circ} 98$, p. 105-128.

GARCIN-MARROu Isabelle, 2009, "Ségolène Royal ou le difficile accès au panthéon politique», Mots, Les langages du politique, nº0, "Présidentielle 2007. Scènes de genre », p.13-29.

GobIN Corinne, 2002, «Le discours programmatique de l'Union européenne. D'une privatisation de l'économie à une privatisation du politique? », Sciences de la société, n'55, p. 156-169.

GoffmAn Erving, 1973, La mise en scène de la vie quotidienne, t. I, La présentation de soi, Paris, Minuit.

Kerbrat-OrReChionI Catherine, 2010, "Pour une analyse multimodale des interactions orales. L'expression des émotions dans les débats politiques télévisuels», Cadernos de Letras da UFF, n ${ }^{40}$, p.17-45.

Kerbrat-OrReChIONI Catherine, 2013, "Animer à deux un débat électoral. L'exemple du duel Royal-Sarkozy (2 mai 2007)», Bulletin suisse de linguistique appliquée, no98, p. 61-81. 
Lallement Michel, SpURK Jan, 2003, Stratégies de la comparaison internationale, Paris, CNRS.

Le BART Christian, 2009, "Les présidentiables de 2007 entre proximité et surplomb. Nicolas Sarkozy et Ségolène Royal vus par Libération », Mots. Les langages du politique, n०89, p. 39-55.

Leroux Pierre, Riutort Philippe, 2014, " “Passer à la télé”. Analyser la présence des professionnels de la politique au sein des émissions conversationnelles», Réseaux. Communication, technologie, société, nº 187, p. 51-77.

LIN HARDT Dominique, MunIESA Fabian, «Du ministère à l'agence. Étude d'un processus d'altération politique », Politix, n²95, p. 73-102.

MANGE Jessica, MARChAN D Pascal, 2011, «Convaincre de ses similitudes tout en démontrant ses différences. Un exemple d'analyse automatique des débats internes au PS », Discours en contextes, L. Baugnet, T. Guilbert éd., Paris, PUF, p. 87-101.

MARChAn D Pascal, 2007, Le grand oral. Les discours de politique générale de la Ve République, Bruxelles, De Boeck / Paris, INA.

Marchand Pascal, Ratinaud Pierre, 2014, "Postures sociodiscursives et conquête du pouvoir », Recherches en communication, n ${ }^{\circ 1}$, p.117-139.

MAYAFFRE Damon, 2003, «Dire son identité politique. Étude du discours politique français au xxe siècle», Cahiers de la Méditerranée, n66, p. 247-264.

MEAD George Herbert, 2006, L'esprit, le soi et la société, Paris, PUF.

OGIEN Albert, 2010, «La valeur sociale du chiffre. La quantification de l'action publique entre performance et démocratie», Revue française de socio-économie, nº 5, p. 19-40.

Oustinoff Michaël, 2010, «Langues de bois d'hier et parler vrai d'aujourd'hui. De la "novlangue" aux "spin doctors" ", Hermès. Cognition, communication, politique, $\mathrm{n}^{\circ} 58$, p. $15-21$.

Planchenault Gaëlle, « Mise en scène du discours d'autorité des experts dans la publicité. De l'éloquence du scientifique à la maladresse du praticien», Mots. Les langages du politique, $\mathrm{n}^{\circ}$ 105, «Couleurs politiques », p. 105-126.

TAJFEL Henri, TURNER John, 1986, «The social identity theory of intergroup behaviour», Psychology of intergroup relations, S. Worchel et W. Austin éd., Chicago, NelsonHall, p. 7-24. 
